\title{
Pharmacotherapy of panic disorder
}

\author{
Charles B Pull' \\ Cristian Damsa ${ }^{2}$ \\ 'Department of Neurosciences, \\ Centre Hospitalier de Luxembourg, \\ Luxembourg; ${ }^{2}$ Department of \\ Psychiatry, Clinical Investigation \\ Program, University of Colorado \\ Health Sciences Center, USA
}

Correspondence: Charles B Pull

Department of Neurosciences, Centre

Hospitalier de Luxembourg, 4 rue Barblé,

I210 GD de Luxembourg

$\mathrm{Tel}+\mathrm{I}+352$ 44I I 2706

Fax + I +352 44I247

Email pull.charles@chl.lu

\begin{abstract}
Panic disorder (PD) is a common, persistent and disabling mental disorder. It is often associated with agoraphobia. The present article reviews the current status of pharmacotherapy for PD with or without agoraphobia as well as the current status of treatments combing pharmacotherapy with cognitive behavior therapy (CBT). The review has been written with a focus on randomized controlled trials, meta-analyses, and reviews that have been published over the past few years. Effective pharmacological treatments include tricyclic antidepressants, monoamine oxidase inhibitors, selective serotonin reuptake inhibitors, serotonin norepinephrine reuptake inhibitors, and various benzodiazepines. Treatment results obtained with CBT compare well with pharmacotherapy, with evidence that CBT is at least as effective as pharmacotherapy. Combining pharmacotherapy with CBT has been found to be superior to antidepressant pharmacotherapy or CBT alone, but only in the acute-phase treatment. Long term studies on treatments combining pharmacotherapy and CBT for PD with or without agoraphobia have found little benefit, however, for combination therapies versus monotherapies. New investigations explore the potential additional value of sequential versus concomitant treatments, of cognitive enhancers and virtual reality exposure therapy, and of education, self management and Internet-based interventions.
\end{abstract}

Keywords: Panic disorder, agoraphobia, pharmacotherapy, cognitive-behavioral therapy, combination treatments.

\section{Clinical presentation}

Panic disorder (PD) is a common, persistent and disabling mental disorder. The efficacy of various psychotropic medications (mainly antidepressants and benzodiazepines) as well as the efficacy of cognitive behavior therapy (CBT) for treating the disorder has been established in numerous randomized controlled trials.

According to the Diagnostic and Statistical Manual of Mental Disorders, Fourth Edition, Text Revision (DSM-IV-TR) (American Psychiatric Association 2000), the essential feature of PD is the presence of recurrent, unexpected panic attacks, followed by at least 1 month of persistent concern about having another panic attack, worry about the possible implications or consequences of the panic attacks, or a significant behavioral change related to the attacks. The panic attacks are not due to the direct physiological effects of a substance or a general medical condition, and are not better accounted for by another mental disorder.

In DSM-IV-TR, a panic attack is defined as a discrete period of intense fear or discomfort accompanied by at least 4 of 13 somatic or cognitive symptoms. The attack has a sudden onset and builds to a peak rapidly (usually in 10 minutes or less) and is often accompanied by a sense of imminent danger or impending doom and an urge to escape. The 13 somatic or cognitive symptoms are (1) palpitations, pounding heart, or accelerated heart rate; (2) sweating; (3) trembling or shaking; (4) sensations or shortness of breath or smothering; (5) feelings of choking; (6) chest pain or discomfort; (7) nausea or abdominal distress; (8) feeling dizzy, unsteady, light-headed, or faint; (9) derealization (feelings of unreality) or depersonalization (being detached from oneself); (10) fear of losing control or going crazy; (11) fear of dying; (12) paresthesias 
(numbness or tingling sensations); and (13) chills or hot flushes. Panic attacks that meet all other criteria but that have fewer than 4 somatic or cognitive symptoms are referred to as limited-symptom attacks.

According to DSM-IV-TR, the essential feature of agoraphobia is anxiety about being in places or situations from which escape might be difficult (or embarrassing) or in which help may not be available in the event of having a panic attack or panic-like symptoms. The anxiety typically leads to a pervasive avoidance of a variety of situations that may include being alone outside the home or being home alone; being in a crowd of people; travelling in a automobile, bus, or airplane; or being on a bridge or in an elevator. Some individuals are able to expose themselves to the feared situations but endure these experiences with considerable dread. Others require the presence of a companion to confront the feared situation. Individuals' avoidance of situations may impair their ability to travel to work or to carry out homemaking responsibilities. According to DSM-IV-TR, agoraphobia may develop at any point in the course of PD, but onset is usually within the first year of occurrence of panic attacks.

There are 3 characteristic types of panic attacks, with different relationships between the attack and the presence or absence of situational triggers: unexpected (uncued) panic attacks, situationally bound (cued) panic attacks, and situationally predisposed panic attacks. Panic attacks can occur in a variety of anxiety disorders, eg, PD, social phobia, specific phobia, acute stress disorder, and post-traumatic stress disorder. In DSM-IV-TR, the occurrence of at least 2 unexpected panic attacks is required for a diagnosis of $\mathrm{PD}$ with or without agoraphobia.

Depending on whether criteria are also met for agoraphobia, PD with agoraphobia or PD without agoraphobia is diagnosed. PD without agoraphobia is characterized by recurrent unexpected panic attacks and by the absence of agoraphobia. PD with agoraphobia is characterized by both recurrent unexpected panic attacks and agoraphobia.

\section{Prevalence}

PD with or without agoraphobia is a highly prevalent psychiatric disorder that affects up to $5 \%$ of the population at some point in life. As shown by the results of the National Comorbidity Survey Replication (Kessler et al 2006), life-time prevalence for PD with agoraphobia is 1.1 and 3.7 for PD without agoraphobia. PD with agoraphobia is diagnosed 3 times as often, and PD without agoraphobia twice as often in women as in men. PD is often disabling, especially when complicated by agoraphobia. The disorder may persist for years and be associated with a considerable impairment in functioning and with a substantial reduction in quality of life. PD with or without agoraphobia is associated with increased use of health care, high rates of absenteeism, and reduced workplace productivity.

\section{Pharmacotherapy}

Medications with proven efficacy in PD include various antidepressants (TCAs), monoamine oxidase (MOA) inhibitors, selective serotonin reuptake inhibitors (SSRIs), serotonin norepinephrine reuptake inhibitors (SNRIs), and benzodiazepines.

\section{Tricyclic antidepressants}

The efficacy of the tricyclic antidepressant imipramine for treating PD was first shown by Donald Klein (1964). After Klein's findings, other tricyclic antidepressants were found to be effective for treating the disorder.

The current use of tricyclic antidepressants for treating PD and other anxiety disorders is addressed by Roy-Byrne and Cowley (2002). While several tricyclic antidepressants are effective treatments for PD with or without agoraphobia, their use is limited by their side effects.

A meta-analysis including 43 studies (Bakker et al 2002) compared the short-term efficacy of selective serotonin reuptake inhibitors vs tricyclic antidepressants in the treatment of PD. Outcome was measured with the proportion of patients becoming panic-free, and with pre/post Cohen's d effect sizes, calculated for four clinical variables: panic, agoraphobia, depression, and general anxiety. There were no differences between SSRIs and TCAs on any of the effect sizes, indicating that both groups of antidepressants are equally effective in reducing panic symptoms, agoraphobic avoidance, depressive symptomatology and general anxiety. In addition, the percentage of patients free of panic attacks at post-test did not differ. The number of drop outs, however, was significantly lower in the group of patients treated with SSRIs. The authors conclude that SSRIs and TCAs are equal in efficacy in the treatment of PD, but SSRIs are tolerated better. In a study by Mavissakalian (2003), imipramine was found to act more rapidly than sertraline, but there was no difference in efficacy between the two drugs at week 8 . Side effects were more frequent with imipramine.

\section{Monoamine oxidase inhibitors}

Benefit and side effects of irreversible MAO inhibitors as well as of reversible ones for treating PD and other disorders have been reported (Riederer et al 2004).

While several irreversible MAO inhibitors are effective treatments for PD with or without agoraphobia, their use is 
limited by their side effects and by their interactions with other drugs and food components. Selective and reversible MAO-A inhibitors (RIMAs) exemplified by moclobemide and brofaromine do not require dietary restrictions, have fewer drug interactions than irreversible MAO inhibitors, and are better tolerated. In 2 comparative trials (Kruger and Dahl 1999; Tiller et al 1999), moclobemide was found to be as efficacious as fluoxetine or clomipramine in the treatment of PD with or without agoraphobia and to have no serious side effects. In 2 comparative trials (Bakish and al 1993; Van Vliet et al 1996), brofaromine was found to be as efficacious as clomipramine or fluvoxamine and to have no serious side effects.

\section{Selective serotonin reuptake inhibitors (Table I)}

During the past 10 years, SSRIs have emerged as first-line pharmacological treatments for PD with or without agoraphobia. Six SSRIs are effective and well-tolerated short term treatments of PD: fluvoxamine, paroxetine, fluoxetine, sertraline, citalopram, and escitalopram.

\section{Fluvoxamine}

In a study by Black and colleagues (1993), patients with moderate to severe PD were randomly assigned to receive 8 weeks of fluvoxamine, cognitive therapy, or placebo. At the main comparison point, $57 \%$ of patients receiving fluvoxamine were rated moderately improved or better vs $40 \%$ of the group given cognitive therapy and $22 \%$ of the placebo-treated group. At that point, $43 \%$ of the fluvoxamine recipients vs $25 \%$ of cognitive therapy and $4 \%$ of placebo recipients were free of panic attacks. Hoehn-Saric et al (1993) compared fluvoxamine with placebo in $50 \mathrm{PD}$ patients, using an 8-week, double-blind, parallel-groups design. At the end of the study, significantly more patients on fluvoxamine were free of major and minor panic attacks than those on placebo. A double-blind, placebo-controlled, multicenter trial involving 54 outpatients (Bakish et al 1996) compared the efficacy, tolerability, and safety of fluvoxamine, imipramine, and placebo in the treatment of PD with or without agoraphobia. Fluvoxamine was more effective than placebo and as effective as imipramine in reducing spontaneous panic attacks in moderate to severe PD. An 8-week multicenter, double-blind, placebo-controlled study in outpatients (Asnis et al 2001) compared fluvoxamine with placebo in patients with DSM-III-R defined PD with or without agoraphobia. Fluvoxamine produced highly significant improvements in

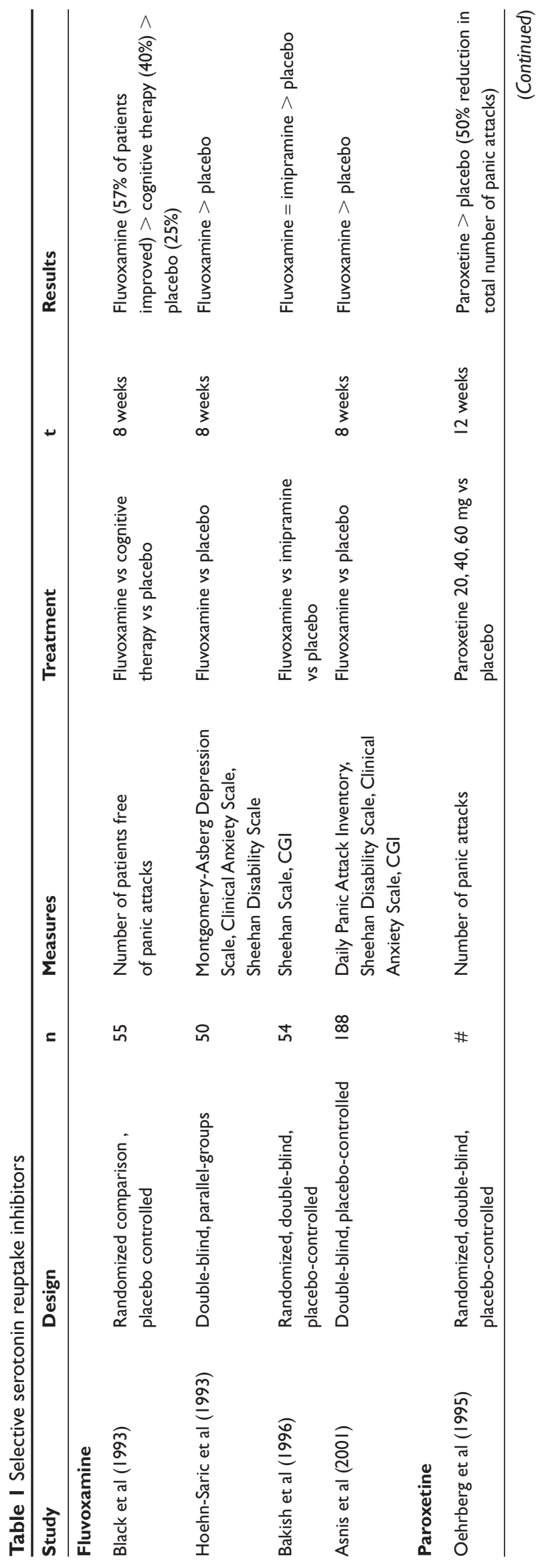




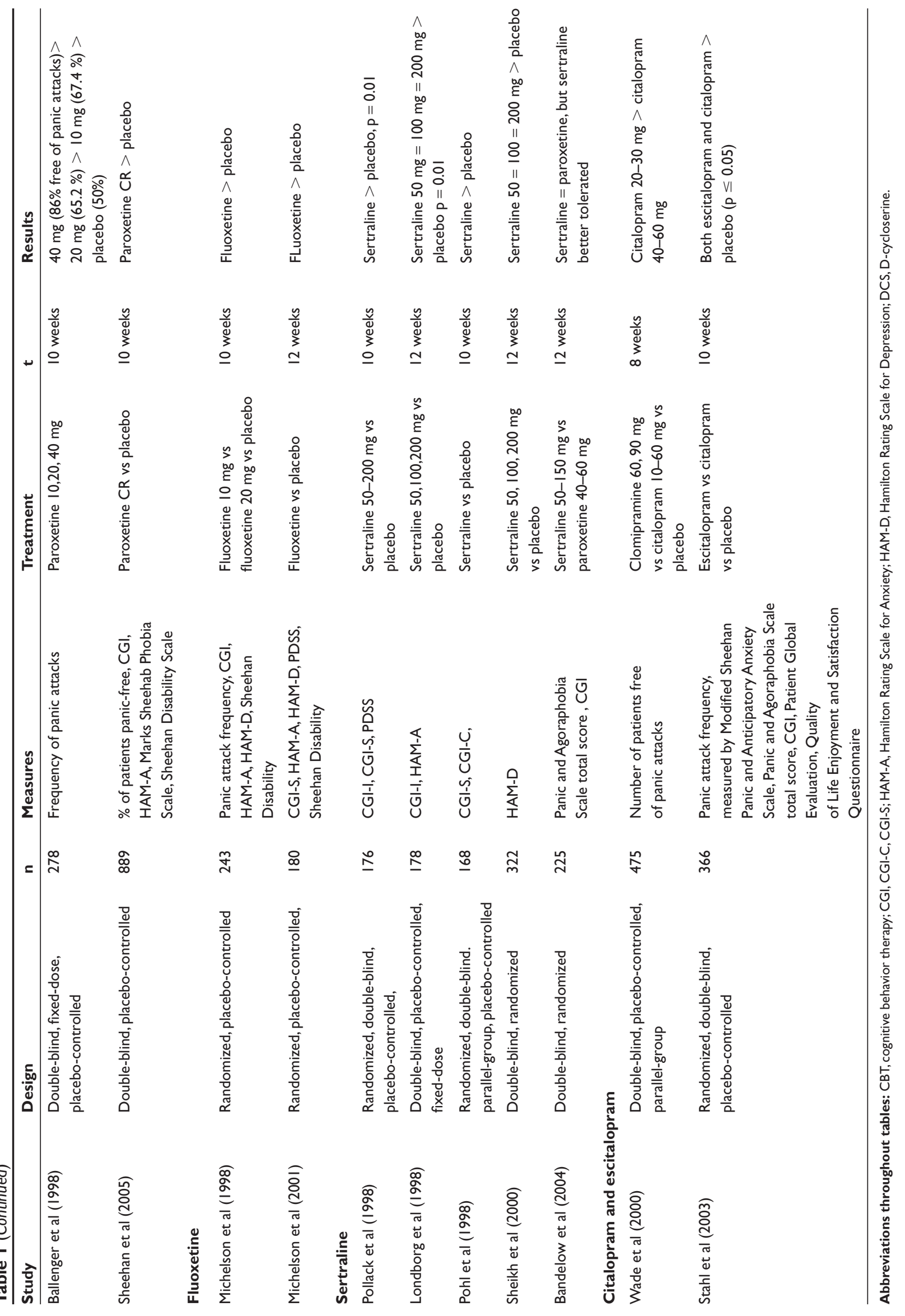


most measures of the frequency and severity of PD and in the more global aspects of disability and distress.

\section{Paroxetine}

In a randomized, double-blind, placebo-controlled study (Oehrberg et al 1995), patients received 12 weeks of treatment with paroxetine $(20,40$, or $60 \mathrm{mg})$ or placebo. Analysis of the results showed statistically significant differences in favor of paroxetine between the two treatment groups in 2 primary measures of outcome, ie, $50 \%$ reduction in total number of panic attacks and number of panic attacks reduced to one or zero over the study period. In a 12-week, double-blind, parallel group, placebo-controlled study, Lecrubier et al (1997) compared paroxetine with clomipramine in 367 patients with DSM-III-R defined PD. Paroxetine produced significant improvements compared with placebo in various measurements of panic attack frequency, and was as effective as clomipramine. In a large, double-blind, fixed-dose, placebocontrolled, multicenter study of paroxetine in the treatment of PD (Ballenger et al 1998), patients were assigned to 10, 20 , or $40 \mathrm{mg} /$ day of study medication. At week $10,86 \%$ of the patients taking $40 \mathrm{mg} / \mathrm{day}, 65.2 \%$ of those taking $20 \mathrm{mg}$, $67.4 \%$ of those taking $10 \mathrm{mg}$, and $50.0 \%$ of the placebo-treated patients were free of full panic attacks.

Sheehan et al (2005) compared controlled-release (CR) paroxetine with placebo in patients with DSM-IV PD with or without agoraphobia in 3 large, identical, double-blind, placebo-controlled, 10-week clinical trials that were pooled for analysis. Paroxetine CR was statistically superior to placebo in the primary outcome measure, ie, percentage of patients who were free of panic attacks in the 2 weeks prior to endpoint. Of the total population that completed or prematurely terminated treatment, $63 \%$ and $53 \%$ of paroxetine CR- and placebo-treated patients, respectively, were panicfree during the final 2 weeks. For week 10 completers (72\% of total), $73 \%$ and $60 \%$ of paroxetine CR- and placebo-treated patients, respectively, were panic-free at week 10 .

\section{Fluoxetine}

In a randomized controlled study of fluoxetine versus placebo (Michelson et al 1998), patients with a diagnosis of PD were randomly assigned to treatment with 10 or 20 $\mathrm{mg}$ /day of fluoxetine or placebo. Fluoxetine, particularly the $20 \mathrm{mg}$ /day dose, was associated with more improvement than was placebo in patients with PD across multiple symptom measures, including global improvement, total panic attack frequency, phobic symptoms, and functional impairment. In another randomized, placebo-controlled trial (Michelson et al 2001), patients with PD were randomized to placebo or to fluoxetine initiated at $10 \mathrm{mg}$ daily for 1 week and then increased to $20 \mathrm{mg}$ daily. The trial lasted 12 weeks, but after 6 weeks patients who had failed to achieve a satisfactory response were eligible for dose escalation to a maximum of $60 \mathrm{mg}$ of fluoxetine daily. The results showed that fluoxetine at a dose of $20 \mathrm{mg}$ daily was associated with a statistically significantly greater proportion of panic-free patients compared with placebo after 6 weeks and at end-point.

\section{Sertraline}

In a flexible-dose multicenter trial (Pollack et al 1998), 176 nondepressed outpatients with PD, with or without agoraphobia, from 10 sites followed identical protocols that used a flexibledose design. Patients were randomly assigned to 10 weeks of double-blind, flexible-dose treatment with either sertraline hydrochloride (50-200 mg) or placebo. Sertraline-treated patients exhibited significantly greater improvement at end point than did patients treated with placebo for panic attack frequency. In a multisite, double-blind, placebo-controlled, fixeddose investigation (Londborg et al 1998), 178 out-patients with PD were randomly assigned to 12 weeks of double-blind treatment with sertraline $(50,100$, or $200 \mathrm{mg})$ or placebo. Sertraline was superior to placebo in reducing the number of panic attacks, situational attacks, unexpected attacks, limited symptom attacks, and time spent worrying. Higher doses were no more effective than the $50 \mathrm{mg}$ dose.

In a randomized, double-blind, parallel-group, flexibledose comparison of sertraline and placebo in outpatients with a DSM-III-R diagnosis of PD with or without agoraphobia (Pohl et al 1998), sertraline was significantly more effective than placebo in decreasing the number of full and limitedsymptom panic attacks. Sheikh and colleagues (2000) pooled data from 2 fixed-dose studies. Patients meeting DSM-III-R criteria for moderate-to-severe PD with or without agoraphobia were randomized sertraline $(50 \mathrm{mg}, 100 \mathrm{mg}$, or $200 \mathrm{mg}$ ). All three sertraline doses produced significant efficacy compared with placebo, with no consistent evidence of a dose-response effect. In a double-blind comparison (Bandelow et al 2004), adult outpatients with PD with or without agoraphobia were randomly assigned to 12 weeks of treatment with flexible doses of sertraline $(50-150 \mathrm{mg})$ or paroxetine $(40-60 \mathrm{mg})$. Sertraline and paroxetine had equivalent efficacy in PD.

\section{Citalopram and escitalopram}

In a double-blind, placebo and clomipramine controlled, parallel group 8-week study (Wade et al 2000), a total of 
475 patients with $\mathrm{PD}$, with or without agoraphobia, were randomized to treatment with either placebo, clomipramine 60 or $90 \mathrm{mg} /$ day, or citalopram 10 or $15 \mathrm{mg} /$ day, or 20 or $30 \mathrm{mg} /$ day, or 40 or $60 \mathrm{mg} /$ day. Treatment with citalopram at 20 or $30 \mathrm{mg}, 40$ or $60 \mathrm{mg}$ and clomipramine were significantly superior to placebo, judged by the number of patients free of panic attacks in the week prior to the final assessment. All rating scales examined suggested that citalopram 20 or $30 \mathrm{mg}$ was more effective than citalopram 40 or $60 \mathrm{mg}$.

A randomized, double-blind, placebo-controlled trial of 366 subjects (128 escitalopram patients, 119 citalopram patients, and 119 placebo patients) conducted by Stahl et al (2003) showed that escitalopram is efficacious, safe, and well tolerated in the treatment of PD. The rate of discontinuation for adverse events was $6.3 \%$ for escitalopram, $8.4 \%$ for citalopram, and $7.6 \%$ for placebo.

\section{Serotonin norepinephrine reuptake inhibitors (Table 2)}

Among the class of SNRIs, venlafaxine and venlafaxine extended-release (ER) have been shown to be effective in the acute treatment of PD with or without agoraphobia. A small 8-week, double-blind, placebo-controlled study (Pollack et al 1996) investigated the efficacy of venlafaxine for the treatment of PD. Patients treated with venlafaxine experienced significantly greater global improvement than those on placebo and exhibited trends toward greater improvement on anxiety and depression symptoms as assessed by the Hamilton rating scales.

In a double-blind, placebo-controlled study (Bradwejn et al 2005), 361 adult out-patients with PD were randomly assigned to receive venlafaxine ER (75-225 mg/day) or placebo for up to 10 weeks in a double-blind study. Venlafaxine ER was not associated with a greater proportion of patients free from full-symptom panic attacks at the final on-therapy evaluation, but was associated with lower mean panic attack frequency and a higher proportion free from limited-symptom panic attacks, higher response and remission rates, and improvements in anticipatory anxiety, fear and avoidance. Pollack et al (2007) compared the efficacy of venlafaxine ER (75 mg/day or $150 \mathrm{mg} /$ day $)$, and paroxetine $(40 \mathrm{mg} /$ day) with placebo in adult outpatients who met DSM-IV criteria for PD with or without agoraphobia. No significant differences were observed between active treatment groups. Panic-free rates at end point with active treatment ranged from $54 \%$ to $61 \%$, compared with $35 \%$ for placebo. Approximately $75 \%$ of patients given

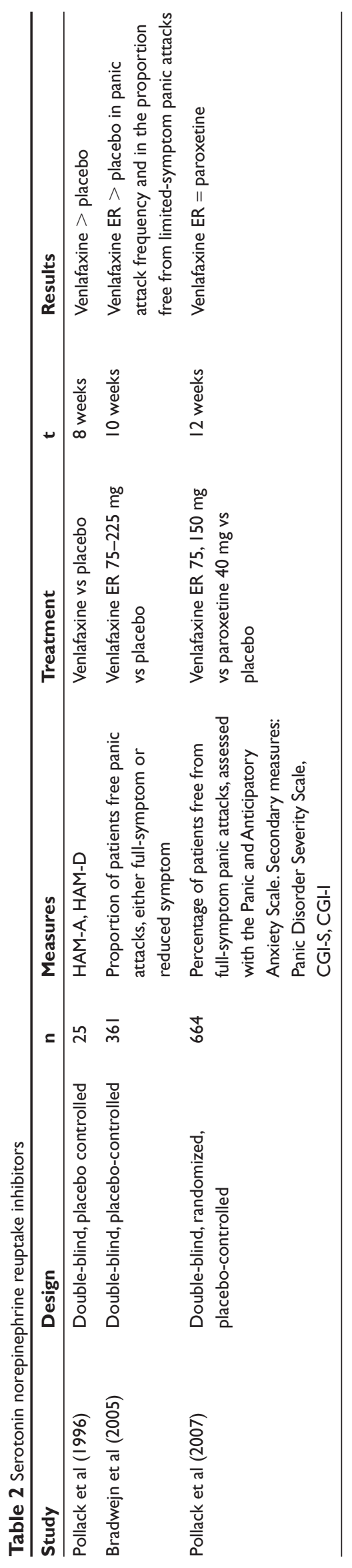


active treatment were responders, and nearly $45 \%$ achieved remission.

\section{Benzodiazepines}

Benzodiazepines such as lorazepam (Schweitzer et al 1990), alprazolam (Verster and Volkerts 2004),) and clonazepam (Rosenbaum 2004) are effective treatments for PD with or without agoraphobia, alone or in combination with one of the SSRIs.

Issues in the clinical use of high-potency benzodiazepines such as lorazepam, alprazolam, and clonazepam for treating PD with or without agoraphobia have been discussed in a review article by Chouinard (2004). Highpotency benzodiazepines are effective in treating PD and panic attacks with or without agoraphobia, alone or as add-on therapy to selective serotonin reuptake inhibitors. Although as a class benzodiazepines act rapidly and are well tolerated, their use presents clinical issues such as dependence, rebound anxiety, memory impairment, and discontinuation syndrome.

As noted by Pollack et al (2003), combining benzodiazepine and antidepressant agents early in treatment offers the potential of accelerating response compared with antidepressant monotherapy and the possibility of additive or synergistic effects from the two classes of agents, but there has been relatively little systematic assessment of the safety and efficacy of this therapeutic strategy. These authors report the results of a randomized, double-blind, 3-arm study in patients with $\mathrm{PD}$, comparing the efficacy and safety of paroxetine and placebo, paroxetine coadministered with clonazepam followed by a tapered benzodiazepine discontinuation phase, and ongoing combination treatment. All treatment groups demonstrated significant improvement by endpoint. There was a significant advantage for the combined treatment groups early in treatment but, subsequently, outcome in all three groups was similar. The combined treatment with paroxetine and clonazepam resulted in more rapid response than with the SSRI alone, but there was no differential benefit beyond the initial few weeks of therapy.

Bearing in mind that pharmacological treatment guidelines for PD have changed as newer treatment options have become available, Bruce et al (2003) examined how the use of psychotropic drugs in patients with PD with or without agoraphobia has shifted over the course of 10 years. A total of 443 patients with PD were interviewed to examine their use of psychotropic medications. Treatment patterns for psychotropic drugs appeared to have remained stable over the past decade, with benzodiazepines being the most commonly used medication for PD. These results highlight a gap between pharmacological treatment guidelines and actual delivery of care.

\section{Long-term pharmacological treatment}

There has been little systematic work done regarding the long-term treatment of PD with or without agoraphobia, and data on the prophylactic effectiveness of maintenance treatment are still limited, as well as information on the risk of relapse due to discontinuation of pharmacological treatment after an initial response to acute therapy.

\section{Maintenance treatment (Table 3)}

Lecrubier and Judge (1997) studied the longer term effects of continuous paroxetine or clomipramine treatment in patients with DSM-III-R defined PD. Patients who satisfactorily completed a 12-week, double-blind, placebo-controlled study of paroxetine and clomipramine could choose to continue receiving their randomized treatment for a further 36 weeks. Paroxetine and clomipramine were significantly more effective than placebo throughout the long-term study in reduction from baseline of full panic attacks, and at the end of treatment in the proportion of patients who eventually experienced no panic attacks. There were no significant differences between paroxetine and clomipramine. Not only was efficacy maintained, but continued improvement was also seen, indicating the importance of long-term treatment in patients with $\mathrm{PD}$.

A controlled, prospective, double-blind, long-term treatment study of citalopram (Lepola et al 1998) followed patients for 1 year. Patients were assigned to 1 of 3 fixed dosage ranges of citalopram, 1 dosage range of clomipramine, or placebo. After an 8-week acute treatment period, patients could continue the treatment for up to 1 year. Of the 475 patients who were randomly assigned for the short-term trial, 279 agreed to continue doubleblind treatment at their assigned doses. In all drug-treated groups, except the group receiving the lowest citalopram dose, the treatment outcome was generally better than with placebo. As determined by a life table analysis of response, the probability of response during the 12 months was significantly greater with all treatment regimens than with placebo, with citalopram 20 or $30 \mathrm{mg} /$ day demonstrating the best response.

In a study by Michelson et al (1999), patients who responded to acute fluoxetine treatment were randomized to 24 weeks of continued fluoxetine or placebo. Fluoxetine 
responders randomized to continue on their acute-phase fluoxetine dose experienced statistically significant improvement in panic attack frequency and phobia rating scale score over 24 weeks of therapy, while those switched to placebo experienced statistically significant worsening in Hamilton Anxiety (HAM-A), Hamilton Depression (HAM-D), and SCL-90-R rating scores.

\section{Discontinuation}

According to Roy-Byrne and Cowley (2002), many studies show clearly that discontinuation of medication results in relapse in a substantial proportion of patients, with rates of $25 \%-50 \%$ recorded within 6 months, depending on study design. With reference to a study by Rickels and colleagues (1993), the same authors state that, in addition, SSRIs, SNRIs, tricyclic antidepressants, and benzodiazepines are associated with a time-limited withdrawal syndrome.

Mavissakalian and Perel (1999) have assessed the 12-month cumulative risk of relapse specifically due to discontinuation of imipramine and tested the hypothesis that maintenance treatment with imipramine protects patients with PD and agoraphobia from such reversals. After an acute-phase open trial with imipramine $(2.25 \mathrm{mg} / \mathrm{kg}$ daily $)$ for 6 months, 56 patients who were in stable remission were randomly assigned to double-blind maintenance or discontinuation conditions. Patients were then followed with planned assessments every 2 months during a 1 -year period. Maintenance treatment (1 relapse) and discontinuation (10 relapses) conditions had significantly different survival curves. The results confirm the very high degree of prophylactic effectiveness of maintenance imipramine treatment and confirm that relapse is substantial after discontinuation of treatment.

Mavissakalian and Perel (2002) further explored the relationship between relapse and duration of imipramine treatment in PD with or without agoraphobia. Fiftyone patients, all in remission at the end of six months acute phase open trial with imipramine $2.25 \mathrm{mg} / \mathrm{kg} / \mathrm{day}$, and randomized to double-blind maintenance or placebo substitution, discontinued imipramine treatment eventually and were followed over a 12-month risk period: 27 during first year placebo substitution, 7 after 12 months of imipramine maintenance in placebo substitution, and 17 after variable durations of imipramine maintenance in open discontinuation. The rate of relapse after only 6 months of treatment ( 10 out of $27,37 \%$ ) was identical to the rate of relapse after 12-30 months of treatment ( 9 out of 24 , $37.5 \%)$. The results suggest a lack of specific protective 
effects beyond prophylaxis and underscore the difficulty in predicting relapse in fully remitted PD with agoraphobia patients.

Two 3-year naturalistic, uncontrolled, non-randomized outcome studies have yielded divergent results. Toni et al (2000) have reported on systematic data from a 3-year naturalistic prospective study on 336 patients with PD with or without agoraphobia treated with antidepressants in a setting of routine clinical practice. The antidepressants used were mainly imipramine, clomipramine, and paroxetine. Of those patients who interrupted the treatment, only $38 \%$ stayed in remission. Relapses after a period of at least 2 months of complete remission were also common, and the probability of presenting at least 1 relapse during the 3-year follow-up period was $67.1 \%$ for PD and 39\% for agoraphobia.

In a study by Dannon et al (2004), 143 patients with PD, with or without agoraphobia, who had responded to a 12-week trial of paroxetine treatment, continued to receive paroxetine maintenance therapy for a total of 12 months or 24 months. After the period of maintenance treatment, patients were monitored throughout a 1-year discontinuation follow-up phase. Only 21 of 143 patients (14\%) relapsed during the 1 -year medication discontinuation follow-up phase. There were no significant differences in relapse rates between the patients who received intermediate-term (12 months) and those who chose the long term course ( 24 months) paroxetine treatment.

\section{Cognitive behavioral therapy and combination treatments}

Cognitive behavioral therapy (CBT) for PD with or without agoraphobia usually combines cognitive restructuring with interoceptive exposure and with progressive exposure to places and situations from which escape might be difficult (or embarrassing), or in which help may not be available in the event of having a panic attack or panic-like symptoms. The efficacy of CBT for the treatment of PD as well as for agoraphobia has been established in a number of randomized controlled trials.

\section{Cognitive behavioral therapy}

In an early meta-analysis of treatment outcome for PD, Gould et al (1995) compared cognitive interventions with exposure treatments and various control conditions. CBT treatments in general had the highest effect size compared with pharmacological treatments or combination treatments. The CBT treatment showed virtually no slippage in effect size by 1-year follow-up compared with sizable slippage for pharmacological treatment. Importantly, there was also a significantly lower attrition rate among CBT treatments compared with pharmacological treatments.

According to 2 recent meta-analyses by Westen et al (2005) and Mitte et al (2005), rates of patients who are panic-free or meet criteria for recovery and high end-state functioning after treatment with CBT compare well with pharmacotherapy, with evidence that CBT is at least as effective as pharmacotherapy, with strong tolerability, maintenance of treatment gains, cost efficacy, improvements in comorbid conditions, and quality of life.

In a recent review, Roy-Byrne et al (2006) concluded that the nature of the evidence concerning the efficacy of CBT is robust, but that CBT approaches are underused in most countries, compared with drug treatment. According to the same authors, low rates of use are probably due to public unfamiliarity with the nature and efficacy of CBT relative to medication, to restricted access to professionals who are familiar with its efficacy and delivery, and to little training and familiarity with CBT for many mental-health professionals who currently treat patients with PD.

The empirical status of CBT for a wide range of disorders, including CBT for PD with or without agoraphobia, has also been reviewed recently by Butler et al (2006). According to the authors, the outcome findings on CBT for PD are supportive and robust. In addition, the authors state that there appears to be robust and convergent metaanalytic evidence that CBT produces vastly superior longterm persistence of effects, with relapse rates half those of pharmacotherapy.

A recent study by Craske et al (2006) raises the possibility that remaining focused on CBT for PD may be more beneficial for both principal and comorbid diagnoses than combining CBT for PD with "straying” to CBT for co-morbid disorders.

\section{Treatments combining pharmacotherapy and CBT}

A substantial proportion of patients does not fully respond or responds poorly to either pharmacotherapy or CBT alone. Since both types of treatment may be effective, it seems reasonable to expect that their combination might enhance the efficacy of either treatment when administered alone. In fact, as pointed out in reviews by Otto et al (2005) and Pull (2006, 2007), empirical data suggest a complex relationship between pharmacotherapy and CBT and highlight the need for more extensive studies, concerning, in particular, the long-term efficacy and effectiveness of combination treatments and the 
effect of discontinuation of either treatment after combined treatment.

Combination treatments for PD with or without agoraphobia have been examined in a number of randomized controlled trials over the past 20 years. Two recent meta-analyses summarize the evidence concerning the short- and long-term benefits and adverse effects of combining pharmacotherapy with CBT versus administrating either treatment alone.

A meta-analysis of the efficacy of psychotherapy and pharmacotherapy in PD with and without agoraphobia by Mitte (2005) included 124 studies. Twenty studies provided data on the efficacy of psychotherapy compared with a combination of psychotherapy and pharmacotherapy. There was a significantly higher effect size for the combination approach for post-test. In contrast, for follow-up (half of the studies included a follow-up), there was no significant difference in efficacy between CBT alone and a combination approach associating CBT and pharmacotherapy.

In a systematic and comprehensive search for relevant trials, Furukava et al (2006) identified 21 studies comparing a combination of psychotherapy (with one exception, the psychotherapy administered in the studies was CBT) and antidepressant pharmacotherapy with either treatment alone. The authors applied the intention-to-treat principle in their analyses (meta-analyses and meta-regressions) by counting all of the drop outs as non-responders. The typical length of the acute-phase active treatment was between 8 and 12 weeks. Nine studies included a follow-up extending over a period of 6-24 months after termination of acute phase and continuation treatments. In the acute phase treatment, the combined therapy was superior to antidepressant pharmacotherapy or psychotherapy alone. After termination of the acute phase treatment, the combined therapy was still more effective than pharmacotherapy alone but no longer more effective than psychotherapy alone. For the comparison between the combination therapy and antidepressant pharmacotherapy, the combination therapy was found to have no disadvantage in the long term, ie, there was no evidence for long-term deleterious effects of combined treatments. For the comparison between combination therapy and psychotherapy, there was no evidence of long-term benefit of the former with the latter, ie, combination treatments were not more effective than CBT alone. The authors conclude that either combined therapy or CBT alone may be chosen as first-line treatment for PD with or without agoraphobia, depending on the patient's preferences.

According to a recent study, the addition of CBT to medication may be associated with less severe side effects and fewer dropouts due to perceived side effects than treatment with medication alone. Marcus et al (2007) assessed whether adding CBT to imipramine for patients with PD decreased the severity of side effects and dropouts from side effects. Data were analyzed for 172 PD patients who were randomly assigned to receive imipramine alone, imipramine plus CBT, or placebo. Patients treated with imipramine plus CBT experienced less severe fatigue/weakness, dry mouth, and sweating and had a lower rate of dropout due to side effects compared with those treated with imipramine only.

\section{Novel combination strategies}

Three novel combination strategies have recently been explored in the treatment of PD with or without agoraphobia: the sequential use of pharmacotherapy and CBT, the combination of virtual reality exposure therapy with $\mathrm{CBT}$, and the use of cognitive enhancers such as d-cycloserine in combination with CBT.

\section{Sequential use of pharmacotherapy and CBT (Table 4)}

Fava et al (2005) have reviewed clinical trials in which treatment components were used in a sequential order, ie pharmacotherapy followed by psychotherapy, psychotherapy followed by pharmacotherapy, one drug treatment after another, or one psychotherapeutic technique after another. According to the authors, the sequential use of pharmacotherapy and CBT introduces a conceptual shift in current treatment approaches of mood and anxiety disorders. It is an intensive, 2-stage approach, which is based on the fact that one course of treatment with a specific treatment (whether pharmacotherapy or psychotherapy) is unlikely to entail the solution to the complex array of symptoms of patients with these disorders.

In the review by Fava et al (2005) the sequential use of pharmacotherapy and psychotherapy was not found to improve long-term outcome in anxiety disorders. In contrast, 2 recent randomized, double-blind, placebo-controlled study do suggest that patients with PD who are unsuccessfully treated with initial CBT may in fact benefit from the addition of an SSRI as a second treatment modality and vice versa.

In a randomized, double-blind, placebo-controlled study Kampman et al (2002) examined the effects of adjunctive paroxetine in PD patients unsuccessfully treated with CBT alone. 161 patients with PD with or without agoraphobia underwent a manual-guided CBT of 15 sessions. Forty-three unsuccessfully treated patients from this group were included in a double-blind, placebo-controlled, next-step treatment study consisting of continued CBT plus adjunctive 


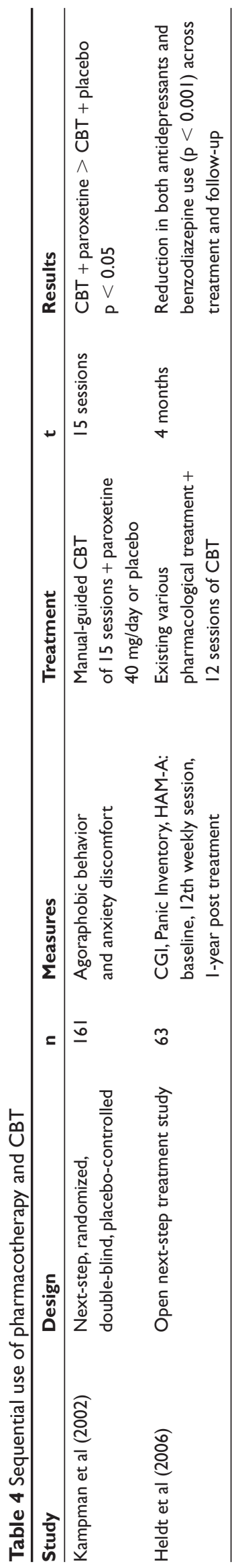

paroxetine at a dose of $40 \mathrm{mg} /$ day or continued CBT plus placebo. Overall, patients in the CBT plus paroxetine condition improved significantly on agoraphobic behavior and anxiety discomfort, whereas patients in the CBT plus placebo condition did not.

Heldt et al (2006) reported the results of an open next-step treatment study involving 63 patients who completed group CBT for PD after failing to respond adequately to previous pharmacotherapy. All patients had been undergoing pharmacological treatment for PD at the time of treatment, with a mean duration of pharmacotherapy of 3 years. In order to be part of the study, patients had to have residual symptoms of PD with or without agoraphobia, such as panic attacks, anticipatory anxiety, and phobic avoidance despite being on a stable dose of medications for at least 4 months. Patients were treated in 1 of 9 groups (with a mean of 7 patients per group) during 12 sessions of CBT provided over 4 months by 2 therapists. Significant reductions in symptoms were evident for all outcome measures (frequency of panic attacks, agoraphobia and anticipatory anxiety) with maintenance of these gains at 1-year follow-up. Follow-up after 1 year showed significant benefit on all dimensional outcome scores, and nearly two-thirds of the sample met remission criteria.

\section{The combination of virtual reality exposure therapy and CBT}

Recent studies provide evidence that virtual reality exposure therapy (VRET) is a promising tool for the treatment of several anxiety disorders. As shown in a review by Pull (2005), the efficacy of standard treatments of anxiety disorders may be significantly enhanced using virtual reality exposure therapy. Most studies in the field have been on specific phobias, in particular on fear of flying, acrophobia, fear of driving, claustrophobia, and fear of spiders. In addition, several studies have been published on the use of virtual reality exposure therapy for social anxiety disorder, posttraumatic stress disorder and PD with or without agoraphobia.

In an ongoing, randomized controlled study by Botella et al (2004a, 2004b), patients with or without agoraphobia are assigned to either VRET, to exposure in vivo, or to a waiting list control group. Initial results of the study indicate that patients in the VRET and in the CBT groups improve significantly more than patients in the waiting list control group, and that there are no significant differences in outcome measures between patients treated by VRET and patients treated by exposure in vivo.

Another ongoing randomized controlled investigation involving the Collège de France and 3 university hospitals 
(in Luxembourg, Lyon, and Paris) compares the efficacy of traditional CBT, VRET, and a waiting list in patients meeting DSM-IV criteria for PD with agoraphobia (Pull et al 2006). CBT and VRET are given in 12 sessions of 90 minutes' duration. Both types of treatment are provided by the same clinicians who are experienced therapists. CBT includes respiratory control, cognitive restructuring, exposure in imagination to anxiety provoking scenes and interoceptive exposure to anxiety-related physical sensations, and homework involving exposure to real-life situations. VR environments are presented using a head mounted display and tracking head movement (Kaiser Electro-Optics Proview $60^{\mathrm{TM}}$, Germany) (Figure 1). VRET includes exposure to 12 virtual environments (taking a subway, walking in a tunnel, taking an elevator, shopping in a supermarket, driving a car on a lonely country road, traveling by plane, entering a movie theatre, driving a car in a city, driving a car in a tunnel, traveling by bus, walking in a crowd, being caught in a sensorial conflict) provoking a feeling of derealization. Initial results of the study indicate that there are no significant differences in outcome measures between patients treated by VRET and patients treated by traditional CBT.

\section{The combination of cognitive enhancers and CBT (Table 5)}

The first cognitive enhancer to be used in addition to CBT in the treatment of anxiety disorders is D-cycloserine (DCS), a partial agonist at the $\mathrm{N}$-methyl-d-aspartate receptor that has previously been shown to improve extinction of fear in rodents. As fear reduction in exposure therapy is similar to extinction learning, DCS has been used recently in 2 investigations, one for the treatment of acrophobia, the other the treatment of social phobia, to determine whether DCS will also improve extinction of fear in human phobic patients undergoing behavioral exposure therapy. The pilot data provide initial support for the use of acute dosing of DCS as an adjunct to exposure-based psychotherapy to accelerate the associative learning processes that contribute to correcting the psychopathology of anxiety disorders.

Ressler et al (2004) enrolled 28 subjects with acrophobia in a randomized, double-blind, placebo-controlled trial

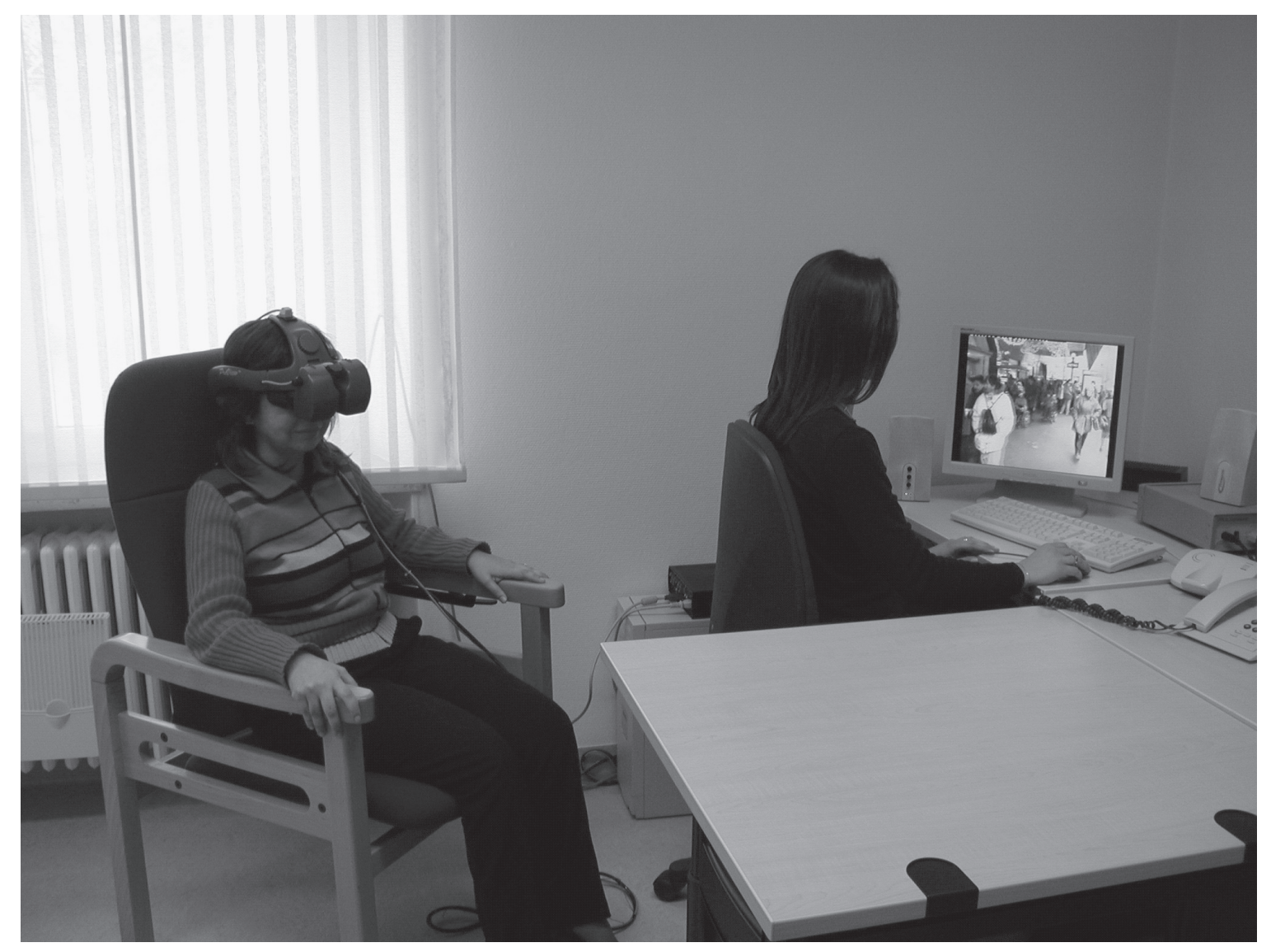

Figure I Virtual reality environments are presented using a head mounted display and tracking head movement. 


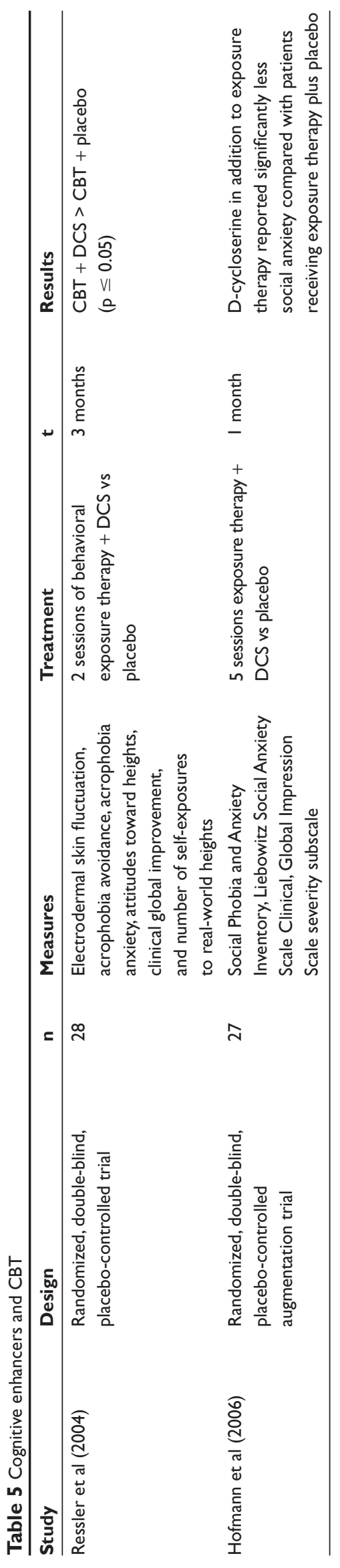

examining DCS versus placebo treatment in combination with a precisely controlled exposure paradigm. Subjects were treated with 2 sessions of behavioral exposure therapy using virtual reality exposure to heights within a virtual glass elevator. Single doses of placebo or DCS were taken prior to each of the 2 sessions of virtual reality exposure therapy. Subjects, therapists, and assessors were blind to the treatment condition. Subjects returned at 1 week and 3 months post-treatment for measures to determine the presence and severity of acrophobia symptoms. Included were measures of acrophobia within the virtual environment, measures of acrophobia in the real world, and general measures of overall improvement. An objective measure of fear, electrodermal skin fluctuation, was also included during the virtual exposure to heights. Symptoms were assessed by self report and by independent assessors at approximately 1 week and 3 months post-treatment.

Exposure therapy combined with DCS resulted in significantly larger reductions of acrophobia symptoms on all main outcome measures. Subjects receiving DCS had significantly more improvement compared with subjects receiving placebo within the virtual environment (1 week after treatment and 3 months later). Subjects receiving DCS also showed significantly greater decreases in post-treatment skin conductance fluctuations during the virtual exposure. Additionally, subjects receiving DCS had significantly greater improvement compared with subjects receiving placebo on general measures of real-world acrophobia symptoms (acrophobia avoidance, acrophobia anxiety, attitudes toward heights, clinical global improvement, and number of self exposures to real world heights). The improvement was evident early in treatment and was maintained at 3 months.

Preliminary support for the use of short-term dosing of d-cycloserine as an adjunctive intervention to exposure therapy has also emerged from a study on social anxiety disorder. In a randomized controlled study by Hofmann et al (2006), participants suffering from social anxiety disorder received 5 therapy sessions delivered in either an individual or group therapy format. The first session provided an introduction to the treatment model and was followed by 4 sessions emphasizing exposure to increasingly challenging public speech situations with videotaped feedback of performances. One hour prior to each session, participants received single doses of d-cycloserine or placebo. Symptoms were assessed before treatment, after treatment, and 1 month after the last session by patient self report and by clinicians blind to the randomization condition. Participants receiving 
d-cycloserine in addition to exposure therapy reported significantly less social anxiety compared with patients receiving exposure therapy plus placebo. Controlled effect sizes were in the medium to large range.

\section{Primary care interventions (Table 6)}

PD with or without agoraphobia is highly prevalent in primary care. During the past few years, several studies have assessed the effectiveness of new treatment approaches in primary care settings. Several interventions, including collaborative care and combined CBT and pharmacotherapy tailored for primary care physicians, have been shown to be more effective than usual care at improving anxiety symptoms, health-related quality of life, and work-related outcomes.

In a randomized controlled study by Rollman et al (2003), patients with PD with or without agoraphobia and patients with generalized anxiety disorder were randomly assigned to a telephone-based care management intervention or to usual care. The intervention involved non-mental health professionals who provided patients with psychoeducation, assessed preferences for guideline-based care, monitored treatment responses, and informed physicians of their patients' care preferences and progress. The usual care patients were informed of their anxiety condition both orally and in a written letter and they were given a disorder-specific brochure on their anxiety diagnosis, but neither the patients nor their physicians were provided with any additional patient-specific treatment advice. At 12-month follow-up, intervention patients reported reduced anxiety and depressive symptoms, improved mental health-related quality of life, larger improvements relative to baseline in hours worked per week, and fewer work days absent in the past month than usual care patients.

In a randomized, controlled study by Roy-Byrne et al (2005), 232 patients were randomized to receive either treatment as usual or an intervention consisting of a combination of up to 6 sessions (across 12 weeks) of CBT modified for the primary care setting, with up to 6 follow-up telephone contacts during the next 9 months, and algorithm-based pharmacotherapy provided by the primary care physician with guidance from a psychiatrist. The combined cognitive behavioral and pharmacotherapeutic intervention resulted in sustained and gradually increasing improvement relative to treatment as usual. The improvement was associated with a moderate increase in ambulatory costs (Katon et al 2006). According to the authors, the additional cost per additional anxiety-free day (AFD) and per quality adjusted life year (QALY) was well within the range of other commonly

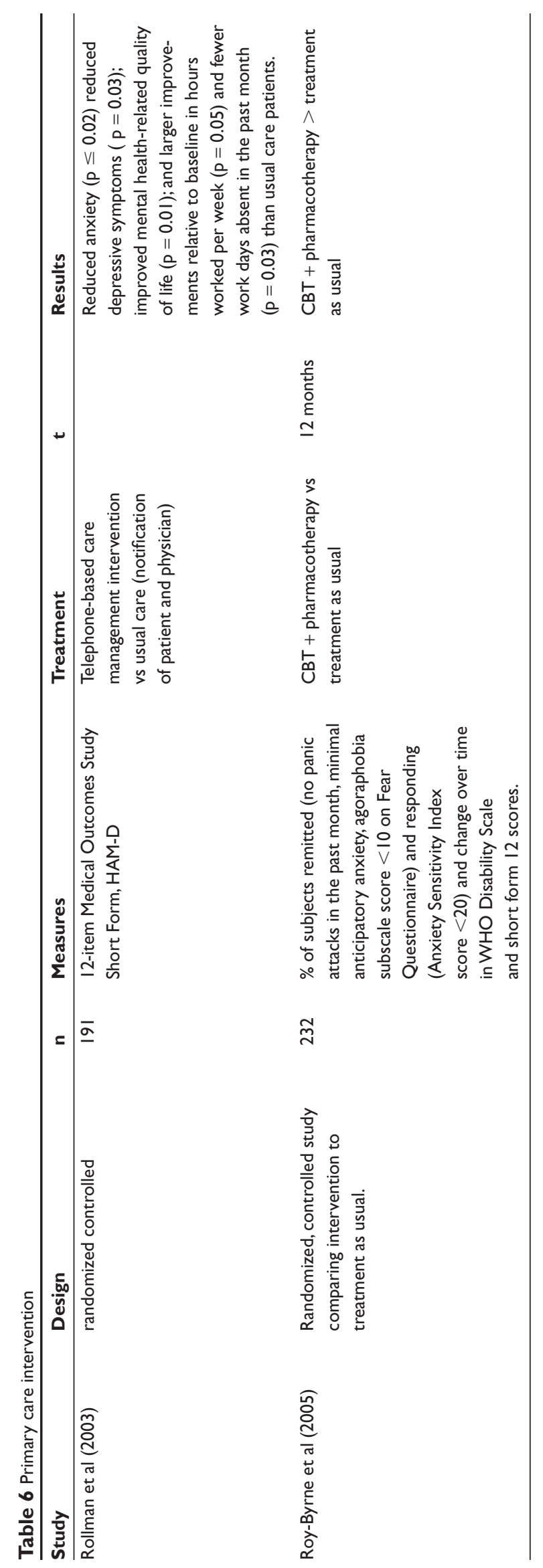


accepted medical interventions such as statin use and treatment of hypertension.

\section{Education, self-management, and Internet-based interventions}

Although PD with or without agoraphobia is often highly amenable to currently available treatments, either pharmacotherapy or CBT, or a combination of both, a considerable proportion of sufferers do not benefit from those treatments, either because they cannot find a specialist to provide them, or because they do not know that such treatments exist. As such, there is a need to bring these treatments to the attention of sufferers and to show them how they can best benefit from them, without or with only limited help by a professional. This is where education, self- management, and Internet-based interventions come in.

Self-management interventions are a set of strategies used by people to manage the symptoms, treatment, and consequences of disorders. Examples of self-help interventions include bibliotherapy, psychoeducation, computer-derived programs, Internet counseling, and peer group interventions. They can be implemented when professional help is either unavailable, or when it is too costly, unwarranted, or not accepted by the patient. Self-help interventions still can involve professionals, but they usually include only limited direct contact with a professional. They rely heavily on the individual to practice self-management techniques. Patients who are intellectually and emotionally capable of understanding the nature of their disorder, as well as the types of treatment options available, and who are willing to invest considerable effort and time in the treatment of their disorder, may greatly profit from such interventions and techniques.

The efficacy of self-management interventions has been investigated in only a limited number of studies, and the methodology in many of those studies is far from perfect. There is, however, sufficient evidence already to support further investigations on self-management interventions for the treatment of PD with or without agoraphobia (Pull 2005).

Calbring et al (2007) have recently published the results of a study that provide evidence to support the use of treatment distributed via the Internet with the addition of short weekly telephone calls to treat PD. The authors evaluated a 10-week Internet-based bibliotherapy self-help program with short weekly telephone calls for people suffering from PD with or without agoraphobia. Sixty participants were randomly assigned to either a wait-listed control group or a multimodal treatment package based on CBT plus minimal therapist contact via email. A 10-minute telephone call was made each week to support each participant. Total mean time spent on each participant during the 10 weeks was 3.9 hours. The participants were required to send in homework assignments before receiving the next treatment module. Analyses were conducted on an intention-to-treat basis, which included all randomly assigned participants. From pre-treatment to post-treatment, all treated participants improved significantly on all measured dimensions (bodily interpretations, maladaptive cognitions, avoidance, general anxiety and depression levels, and quality of life). Treatment gains on self-report measures were maintained at the 9 month follow-up. A blind telephone interview after the end of treatment revealed that $77 \%$ of the treated patients no longer fulfilled the criteria for PD, whereas all of the waitlisted subjects still suffered from it.

\section{Conclusion}

Different pharmacological treatments as well as CBT are effective in the acute as well as in the long term treatment of PD with or without agoraphobia. First-line acute pharmacological treatments include different SSRIs such as paroxetine, fluoxetine, fluvoxamine, sertraline, citalopram, and escitalpram as well as the SNRI venlafaxine.

Other medications with proven efficacy in PD include tricyclic antidepressants, MAO inhibitors, and benzodiazepines. While several tricyclic antidepressants are effective treatments for PD with or without agoraphobia, their use is limited by their side effects. While irreversible MAO inhibitors are effective treatments for PD with or without agoraphobia, their use is limited by their side effects and by their interactions with other drugs and food components. Selective and RIMAs exemplified by moclobemide and brofaromine, do not require dietary restrictions, have fewer drug interactions than irreversible MAO inhibitors, and are better tolerated. Highpotency benzodiazepines such as lorazepam, alprazolam, and clonazepam are effective in treating PD and panic attacks with or without agoraphobia, alone or as add-on therapy to SSRIs. Although, as a class, benzodiazepines act rapidly and are well tolerated, their use presents clinical issues such as rebound anxiety, memory impairment, discontinuation syndrome, and a potential for abuse and dependence.

In long-term treatments with several medications, efficacy was maintained or further increased in the long run, indicating the importance of long-term pharmacological treatment. Discontinuation of medication may result in relapse in a substantial proportion of patients.

CBT is a highly effective treatment for PD with or without agoraphobia that compares favorably with any of the 
pharmacological treatments in the acute phase of the treatment and may in fact be more effective in the long run.

Combining pharmacotherapy and CBT may be associated with greater overall efficacy in the acute phase of the treatment than either treatment alone. In the long-term treatment of PD with or without agoraphobia, combination treatments may be more effective than pharmacotherapy alone, but not more effective than CBT alone.

New strategies for combining pharmacotherapy and CBT, such as the sequential administration of pharmacotherapy and CBT, the combination of virtual reality exposure therapy and CBT, or the use of cognitive enhancers in combination with CBT have yielded promising results and should be investigated more fully in the years to come.

Finally, collaborative care strategies tailored for use in primary care as well as self-management interventions are more effective than usual care and should be further developed and refined.

\section{Disclosure}

The authors report no conflict of interest.

\section{References}

American Psychiatric Association. 2000. Diagnostic and statistical manual of mental disorders, IVth edn, Text Revision (DSM-IV-TR). Washington, DC: American Psychiatric Association.

Asnis GM, Hameedi FA, Goddard AW, et al. 2001. Fluvoxamine in the treatment of panic disorder: a multi-center, double-blind, placebocontrolled study in outpatients. Psychiatry Res, 103:1-14.

Bakish D, Hooper CL, Filteau MJ, et al. 1996. A double-blind placebocontrolled trial comparing fluvoxamine and imipramine in the treatment of panic disorder with or without agoraphobia. Psychopharmacoly Bull, 32:135-41.

Bakish D, Saxena BM, Bowen R, et al. 1993. Reversible monoamine oxidase-A inhibitors in panic disorder. Clin Neuropharmacology, 16:77-82.

Bakker A, van Balkom AJ, Spinhoven P. 2002. SSRIs vs TCAs in the treatment of panic disorder: a meta-analysis. Acta Psychiatrica Scand, 106:163-7.

Ballenger JC, Wheadon DE, Steiner M, et al. 1998. Double-blind, fixeddose, placebo-controlled study of paroxetine in the treatment of panic disorder. Am J Psychiatry, 155:36-42.

Bandelow B, Behnke K, Lenoir S, et al. 2004. Sertraline versus paroxetine in the treatment of panic disorder: an acute, double-blind noninferiority comparison. J Clin Psychiatry, 65:405-13.

Black DW, Wesner R, Bowers W, et al. 1993. A comparison of fluvoxamine, cognitive therapy, and placebo in the treatment of panic disorder. Arch Gen Psychiatry, 50:44-50.

Botella C, Villa H, Garcia-Palacios A, et al. 2004a. The use of virtual reality in the treatment of panic and agoraphobia. In: G. Riva, C. Botella, P. Légeron, G. Optale (Eds.). Cybertherapy: Internet and virtual reality as assessment and rehabilitation tools for clinical psychology and neuroscience. Amsterdam: Ios Press.

Botella C, Villa H, Garcia Palacios A, et al. 2004b. The use of VR in the treatment of panic disorders and agoraphobia. Stud Health Techn Informatics, 99:73-90.
Bradwejn J, Ahokas A, Stein DJ, et al. 2005. Venlafaxine extended-release capsules in panic disorder: flexible-dose, double-blind, placebo-controlled study. Br J Psychiatry, 187:352-9.

Bruce SE, Vasile RG, Goisman RM et al. 2003. Are benzodiazepines still the medication of choice for patients with panic disorder with or without agoraphobia? Am J Psychiatry, 160:1432-38.

Butler AC, Chapman JE, Forman EM, et al. 2006. The empirical status of cognitive-behavioural therapy: a review of meta-analyses. Clin Psychol Rev, 26:17-31.

Carlbring P, Bohman S, Brunt S, et al. 2006. Remote treatment of panic disorder: a randomized trial of internet-based cognitive behavior therapy supplemented with telephone calls. Am J Psychiatry, 163:2119-25.

Chouinard G. 2004. Issues in the clinical use of benzodiazepines: potency, withdrawal, and rebound. J Clin Psychiatry, 65:7-12.

Craske MG, Farchione TJ, Allen LB, et al. 2006. Cognitive behavioural therapy for panic disorder and comorbidity: More of the same or less of more? Behav Res Ther, 45:1095-109.

Dannon PN, Iancu I, Cohen A, et al. 2004. Three year naturalistic outcome study of panic disorder patients treated with paroxetine. $B M C$ Psychiatry, 4:16.

Fava GA, Ruini C, Rafanelli C. 2005. Sequential treatment of mood and anxiety disorders. J Clin Psychiatry, 66:1392-400.

Furukawa TA, Watanabe N, Churchill R. 2006. Psychotherapy plus antidepressant for panic disorder with or without agoraphobia: Systematic review. Br J Psychiatry, 188:305-12.

Gould RA, Otto MW, Pollack MH. 1995. A meta-analysis of treatment outcome for panic disorder. Clin Psychol Review, 15:819-44.

Heldt E, Gus Manfro G, Kipper L, et al. 2006.One-year follow-up of pharmacotherapy-resistant patients with panic disorder treated with cognitive-behavior therapy: Outcome and predictors of remission. Behav Res Therapy, 44:657-65.

Hoehn-Saric R, McLeod DR, Hipsley PA. 1993. Effect of fluvoxamine on panic disorder. J Clin Psychopharmacology, 13:321-6.

Kampman M, Keijsers GPJ, Hoogduin CAL, et al. 2002. A randomized, double-blind, placebo-controlled study of the effects of adjunctive paroxetine in panic disorder patients unsuccessfully treated with cognitive-behavioural therapy alone. J Clin Psychiatry, 63:772-7.

Katon W, Russo J, Sherbourne C, et al. 2006. Incremental cost-effectiveness of a collaborative care intervention for panic disorder. Psychological Medicine, 36:353-63.

Kessler RC, Chiu WT, Jin R, et al. 2006. The epidemiology of panic attacks, panic disorder, and agoraphobia in the National Comorbidity Survey Replication. Arch Gen Psychiatry, 63:415-4.

Klein DF. 1964. Delineation of two drug-responsive anxiety syndromes. Psychopharmacologia, 5:397-408.

Kruger MB, Dahl AA. 1999. The efficacy and safety of moclobemide compared to clomipramine in the treatment of panic disorder. Eur Arch Psych Clin Neurosciences, 249:19-24.

Lecrubier Y, Bakker A, Dunbar G, et al. 1997. A comparison of paroxetine, clomipramine and placebo in the treatment of panic disorder. Collaborative Paroxetine Panic Study Investigators. Acta Psychiatr Scand, 95:145-52.

Lecrubier Y, Judge R. 1997. Long-term evaluation of paroxetine, clomipramine and placebo in panic disorder. Collaborative Paroxetine Panic Study Investigators. Acta Psychiatr Scand, 95:153-60.

Lepola UM, Wade AG, Leinonen EV, et al. 1998. A controlled, prospective, 1-year trial of citalopram in the treatment of panic disorder. J Clin Psychiatry, 59:528-34.

Londborg PD, Wolkow R, Smith WT, et al. 1998. Sertraline in the treatment of panic disorder. A multi-site, double-blind, placebo-controlled, fixeddose investigation. Br J Psychiatry, 173:54-60.

Marcus SM, Gorman J, Shear MK, et al. 2007. A comparison of medication side effect reports by panic disorder patients with and without concomitant cognitive behavior therapy. Am J Psychiatry, 164:273-5. 
Mavissakalian MR. 2003. Imipramine vs. sertraline in panic disorder: 24-week treatment completers. Ann Clin Psychiatry, 15:171-80.

Mavissakalian MR, Perel JM. 1999. Long-term maintenance and discontinuation of imipramine therapy in panic disorder with agoraphobia. Arch Gen Psychiatry, 56:821-7.

Mavissakalian MR, Perel JM. 2002. Duration of imipramine therapy and relapse in panic disorder with agoraphobia. J Clin Psychopharmacol, 22:294-9.

Michelson D, Allgulander C, Dantendorfer K, et al. 2001. Efficacy of usual antidepressant dosing regimens of fluoxetine in panic disorder: randomised, placebo-controlled trial. Br J Psychiatry, 179:514-8.

Michelson D, Lydiard RB, Pollack MH, et al. 1998. Outcome assessment and clinical improvement in panic disorder: evidence from a randomized controlled trial of fluoxetine and placebo. The Fluoxetine Panic Disorder Study Group. Am J Psychiatry, 155:1570-7.

Michelson D, Pollack M, Lydiard RB, et al. 1999. Continuing treatment of panic disorder after acute response: randomised, placebo-controlled trial with fluoxetine. The Fluoxetine Panic Disorder Study Group. Br J Psychiatry, 174:213-8.

Mitte K. 2005. A meta-analysis of the efficacy of psycho-and pharmacotherapy in panic disorder with and without agoraphobia. $J$ Affect Disord, 88:27-45.

Oehrberg S, Christiansen PE, Behnke K, et al. 1995. Paroxetine in the treatment of panic disorder. A randomised, double-blind, placebo-controlled study. Br J Psychiatry, 167:374-9.

Otto MW, Powers M, Smits JASD. 2005. Adding cognitive-behavioural therapy to pharmacotherapy for panic disorder: issues and strategies. CNS Spectrums, 9:32-9.

Pohl RB, Wolkow RM, Clary CM. 1998. Sertraline in the treatment of panic disorder: a double-blind multicenter trial. Am J Psychiatry, 155:1189-95.

Pollack MH, Lepola U, Koponen H, et al. 2007. A double-blind study of the efficacy of venlafaxine extended-release, paroxetine, and placebo in the treatment of panic disorder. Depress Anxiety, 24:1-14.

Pollack MH, Otto MW, Worthington JJ, et al. 1998. Sertraline in the treatment of panic disorder: a flexible-dose multicenter trial. Arch Gen Psychiatry, 55:1010-16.

Pollack MH, Simon NM, Worthington JJ, et al. 2003. Combined paroxetine and clonazepam treatment strategies compared to paroxetine monotherapy for panic disorder. J Psychopharmacology, 17:276-82.

Pollack MH, Worthington JJ 3rd, Otto MW, et al. 1996. Venlafaxine for panic disorder: results from a double-blind, placebo-controlled study. Psychopharmacol Bull, 32:667-70.

Pull CB, Cottraux J, Berthoz A, et al. 2006. Randomized controlled study of virtual reality exposure therapy and cognitive-behaviour therapy in panic disorder with agoraphobia. Cyberpsychol Behav, 9:712.

Pull CB. 2005. Current status of virtual reality exposure therapy in anxiety disorders: editorial review. Curr Opin Psychiatry, 18:7-14.

Pull CB. 2006. Self-help interventions for the treatment of anxiety disorders. Curr Opin Psychiatry, 19:50-3.

Pull CB. 2007. Combined pharmacotherapy and cognitive-behavioural therapy for anxiety disorders. Curr Opin Psychiatry, 20:30-5.

Ressler KJ, Rothbaum BO, Tannenbaum L, et al. 2004. Cognitive Enhancers as Adjuncts to Psychotherapy. Use of D-Cycloserine in Phobic Individuals to Facilitate Extinction of Fear. Arch Gen Psychiatry, 61:1136-44.
Rickels K, Schweizer E, Weiss S, et al. 1993. Maintenance drug treatment for panic disorder. II Short- and long-term outcome after drug taper. Arch Gen Psychiatry, 50:61-8.

Riederer P, Lachenmayer L, Laux G. 2004. Clinical applications of MAOinhibitors. Curr Med Chem, 11:2033-43.

Rollman BL, Belnap BH, Mazumdar S, et al. 2003. A randomized trial to improve the quality of treatment for panic and generalized anxiety disorders in primary care. Arch Gen Psychiatry, 62:1332-41.

Rosenbaum JF. 2004. The development of clonazepam as a psychotropic: the massachusetts general hospital experience. J Clin Psychiatry, 65:3-6.

Roy-Byrne PP, Cowley DS. 2002. Pharmacologic treatment for panic disorder, generalized anxiety disorder, specific phobia and social anxiety disorders. In: Nathan P, Gorman JM (eds). A guide to treatments that work. Second Edition. New York: Oxford University Press, pp. 337-65.

Roy-Byrne PP, Craske MG, Stein MB, et al. 2005. A randomized effectiveness trial of cognitive-behavioural therapy and medication for primary care panic disorder. Arch Gen Psychiatry, 62:290-8.

Roy-Byrne PP, Craske MG, Stein MB. 2006. Panic disorder. Lancet, 368:1023-32.

Schweizer E, Pohl R, Balon R, et al. 1990. Lorazepam vs. alprazolam in the treatment of panic disorder. Pharmacopsychiatry, 23:90-3.

Sheehan DV, Burnham DB, Iyengar MK, et al. 2005. Efficacy and tolerability of controlled-release paroxetine in the treatment of panic disorder. J Clin Psychiatry, 66:34-40.

Sheikh JI, Londborg P, Clary CM, et al. 2000. The efficacy of sertraline in panic disorder: combined results from two fixed-dose studies. Int Clin Psychopharmacol, 15:335-42.

Stahl SM, Gergel I, Li D. 2003. Escitalopram in the treatment of panic disorder: a randomized, double-blind, placebo-controlled trial. J Clin Psychiatry, 64:1322-7.

Tiller JW, Bouwer C, Behnke K. 1999. Moclobemide and fluoxetine for panic disorder. International Panic Disorder Study Group. Eur Arch Psych Clin Neurosciences, 249:7-10.

Toni C, Perugi G, Frare F, et al. 2000. A prospective naturalistic study of 326 panic-agoraphobic patients treated with antidepressants. Pharmacopsychiatry, 33:121-31.

Van Vliet IM, den Boer JA, Westenberg HG, et al. 1996. A double-blind comparative study of brofaromine and fluvoxamine in outpatients with panic disorder. J Clin Psychopharmacol. 16:299-306.

Verster JC, Volkerts ER. 2004. Clinical pharmacology, clinical efficacy, and behavioural toxicity of alprazolam: a review of the literature. CNS Drug Rev, 10:45-76.

Wade AG, Lepola U, Koponen HJ, et al. 1997. The effect of citalopram in panic disorder. Br J Psychiatry, 170:549-53.

Westen D, Morrison K. 2001. A multidimensional meta-analysis of treatments for depression, panic, and generalized anxiety disorder: an empirical examination of the status of empirically supported therapies. J Consult Clin Psychol, 69:875-99. 
